\title{
Fractional coloring methods with applications to degenerate graphs and graphs on surfaces
}

\section{Gimbel, John; Kündgen, André; Li, Binlong; Thomassen, Carsten}

\section{Published in:}

SIAM Journal on Discrete Mathematics

Link to article, DOI:

10.1137/18M1177317

Publication date:

2019

Document Version

Peer reviewed version

Link back to DTU Orbit

Citation (APA):

Gimbel, J., Kündgen, A., Li, B., \& Thomassen, C. (2019). Fractional coloring methods with applications to degenerate graphs and graphs on surfaces. SIAM Journal on Discrete Mathematics, 33(3), 1415-1430. https://doi.org/10.1137/18M1177317

\section{General rights}

Copyright and moral rights for the publications made accessible in the public portal are retained by the authors and/or other copyright owners and it is a condition of accessing publications that users recognise and abide by the legal requirements associated with these rights.

- Users may download and print one copy of any publication from the public portal for the purpose of private study or research.

- You may not further distribute the material or use it for any profit-making activity or commercial gain

- You may freely distribute the URL identifying the publication in the public portal 


\title{
FRACTIONAL COLORING METHODS WITH APPLICATIONS TO DEGENERATE GRAPHS AND GRAPHS ON SURFACES*
}

\author{
JOHN GIMBEL ${ }^{\dagger}$, ANDRÉ KÜNDGEN $^{\ddagger}$, BINLONG LI§ ${ }^{\S}$ AND CARSTEN THOMASSEN
}

\begin{abstract}
We study methods for finding strict upper bounds on the fractional chromatic number $\chi_{f}(G)$ of a graph $G$. We illustrate these methods by providing short proofs of known inequalities in connection with Grötzsch's 3-color theorem and the Five Color Theorem for planar graphs. We also apply it to $d$-degenerate graphs and conclude that every $K_{d+1}$-free $d$-degenerate graph with $n$ vertices has independence number $<n /(d+1)$. We show that for each surface $S$ and every $\varepsilon>0$, the fractional chromatic number of any graph embedded on $S$ of sufficiently large width (depending only on $S$ and $\varepsilon$ ) is at most $4+\varepsilon$. In the same spirit we prove that Eulerian triangulations or triangle-free graphs of large width have $\chi_{f} \leq 3+\varepsilon$, and quadrangulations of large width have $\chi_{f} \leq 2+\varepsilon$. While the $\varepsilon$ is needed in the latter two results, we conjecture that in the first result $4+\varepsilon$ can be replaced by 4 . The upper bounds $\chi_{f} \leq 4+\varepsilon, \chi_{f} \leq 3+\varepsilon, \chi_{f} \leq 2+\varepsilon$, respectively, are already known for graphs on orientable surfaces, but our results are also valid for graphs on nonorientable surfaces.

Surprisingly, a strict lower bound on the fractional chromatic number may imply an upper bound on the chromatic number: Grötzsch's theorem implies that every 4-chromatic planar graph $G$ has fractional chromatic number $\chi_{f}(G) \geq 3$. We conjecture that this inequality is always strict and observe that this implies the Four Color Theorem for planar graphs.
\end{abstract}

Key words. Fractional chromatic number, planar graphs, degeneracy, graphs on surfaces

AMS subject classifications. 05C $72,05 \mathrm{C} 15,05 \mathrm{C} 07,05 \mathrm{C} 10$

1. Introduction. The fractional chromatic number $\chi_{f}(G)$ for a graph $G$ is a natural generalization of its chromatic number $\chi(G)$ and has the additional advantage that it can be investigated by linear programming methods, as described for example in the monograph by Scheinerman and Ullman [21]. Linear programming can provide lower bounds for $\chi_{f}(G)$ for specific graphs $G$, but general lower bounds on $\chi_{f}(G)$ tend to be difficult to find, except that $\chi_{f}(G)$ is bounded below by the clique number $\omega(G)$ and also by $|V(G)| / \alpha(G)$, where $\alpha(G)$ is the independence number. The purpose of this paper is to offer some conjectures and give some methods for upper bounds by proving the fractional chromatic number to be strictly smaller than the chromatic number for graphs satisfying well know upper bounds on the chromatic number. As the two chromatic numbers coincide for graphs whose chromatic number equals the clique number, we focus on graphs with no large cliques, in particular triangle-free graphs and graphs with a large width embedding on a surface.

Let $k$ be a positive real number. A graph $G$ is fractionally $k$-colorable if $\chi_{f}(G) \leq k$. A graph $G$ is $k$-fraction-critical if $\chi_{f}(G) \geq k$, but $\chi_{f}(H)<k$ for every one of its proper subgraph $H$. We say that a family of graphs $\mathcal{F}$ is fractionally $k$-colorable if for every $G \in \mathcal{F}$ we have $\chi_{f}(G) \leq k$, and $\mathcal{F}$ is fractionally $(<k)$-colorable if for every $G \in \mathcal{F}$

Funding: This work was done when the first three authors visited the Technical University of Denmark during the first half of 2017. Li was supported by National Natural Science Foundation of China (No. 11601429 \& 11671320). Gimbel, Kündgen and Thomassen were supported by the ERC Advanced Grant GRACOL, project no. 320812.

${ }^{\dagger}$ Department of Mathematics and Statistics, University of Alaska Fairbanks, Fairbanks, AK 99775, United States of America.

${ }_{\ddagger}^{\ddagger}$ Department of Mathematics, California State University San Marcos, San Marcos, CA 92096, United States of America.

$\S$ Department of Applied Mathematics, Northwestern Polytechnical University,Xi'an, Shaanxi 710072, P.R. China.

IDepartment of Applied Mathematics and Computer Science, Technical University of Denmark, DK-2800 Lyngby, Denmark. 
we have $\chi_{f}(G)<k$.

King, Lu and Peng [13] strengthened Brooks' theorem for fractional coloring by proving that a connected graph of maximum degree $d$ containing no $K_{d}$ has $\chi_{f}(G) \leq$ $d-\frac{2}{67}$ unless $G$ is either the square of an 8-cycle $C_{8}^{2}$ or the strong product of $C_{5}$ and $K_{2}$. As another example, Hilton, Rado and Scott [11] showed that the Five Color Theorem for planar graphs can be extended to show that planar graphs are fractionally $(<5)$-colorable. The Four Color Theorem shows that planar graphs are fractionally 4 -colorable, but we do not know if planar graphs are fractionally $(<4)$-colorable with finitely many minimal exceptions (see also Example 3.9 and Conjecture 3.7 ).

In this paper we focus on families of $k$-colorable graphs that are fractionally $(<k)$ colorable. To illustrate the usefulness of a sharpened upper bound on the fractional chromatic number consider a $d$-degenerate graph $G$ with $n$ vertices. Such a graph has chromatic number at most $d+1$ and hence independence number at least $n /(d+1)$. For the complete graph with $d+1$ vertices these bounds are sharp. However, if the graph does not contain a complete graph with $d+1$ vertices, then we prove that the fractional chromatic number is strictly less than $d+1$ and hence it has independence number $\alpha(G)>n /(d+1)$, a fact that does not follow from the chromatic number, which may still be $d+1$. In particular, if $G$ is planar 3-degenerate and contains no $K_{4}$, then $\chi_{f}(G)<4$ even though possibly $\chi(G)=4$. Combining this with the result of Thomassen [28] that every planar graph has an independent set $A$ so that $G-A$ is 3 -degerate gives a new proof (without using the Four Color Theorem) of the result of Hilton, Rado and Scott [11] that planar graphs are fractionally $(<5)$-colorable. (As it suffices to prove this result for planar triangulations with no separating triangle, we may assume that $G-A$ above contains no $K_{4}$.)

We give a simple proof of a fractional version of Grötzsch's 3-color theorem by showing that planar triangle-free graphs are fractionally $(<3)$-colorable, as first proved by Dvořák, Sereni and Volec [5]. In [5] it is conjectured that planar graphs of girth 5 are fractionally $(3-\varepsilon)$-colorable for some fixed positive constant $\varepsilon$, whereas examples by Pirnazar and Ulman [19] (attributed by them to Fraughnaugh) show that this is not the case for planar graphs of girth 4 . Those examples also imply that $d$-degenerate graphs are generally not fractionally $(d+1-\varepsilon)$-colorable for any fixed positive $\varepsilon$. We do not know if 3 -degenerate planar graphs are fractionally $(4-\varepsilon)$ colorable (with finitely many minimal exceptions) for some fixed positive $\varepsilon$.

For surfaces of higher genus we prove that the fractional chromatic number of a graph of large width on a surface is at most $4+\varepsilon$. In the same spirit we prove that Eulerian triangulations of large width and triangle-free graphs of large width are fractionally $(3+\varepsilon)$-colorable, and that quadrangulations of large width are fractionally $(2+\varepsilon)$-colorable. While the $\varepsilon$ is needed in the latter two results, we conjecture that in the first result $4+\varepsilon$ can be replaced by 4 . The upper bounds $\chi_{f} \leq 4+\varepsilon, \chi_{f} \leq 3+\varepsilon$, $\chi_{f} \leq 2+\varepsilon$, respectively, are already proved, even for the circular chromatic number (which is greater than or equal to the fractional chromatic number), for graphs on orientable surfaces by DeVos, Goddyn, Mohar, Vertigan, and Zhu [4]. But, those inequalities do no hold for the circular chromatic number of graphs on nonorientable surfaces, whereas our results for the fractional chromatic number do.

Finally, we prove that graphs of girth at least 5 and large width are fractionally $(<3)$-colorable. Perhaps 3 can be replaced by $(3-\varepsilon)$ with a fixed positive $\varepsilon$ for each surface. For planar graphs of girth 5 it is not possible to replace 3 by any number less than $11 / 4$, as shown by Pirnazar and Ulman [19]. 
2. Basic notation and the chromatic gap. For an introduction to fractional coloring see the book of Scheinerman and Ullman [21].

If $\mathcal{I}$ is the collection of independent sets of a graph $G$, then a function $f: \mathcal{I} \rightarrow$ $[0, \infty)$ is called a fractional coloring of $G$ if for each vertex $v$ the weight $w_{f}(v)=$ $\sum_{I \in \mathcal{I}, v \in I} f(I)$ satisfies $w_{f}(v) \geq 1$. The (total) weight of a fractional coloring $f$ is $w(f)=\sum_{I \in \mathcal{I}} f(I)$, and the minimum such weight over all possible fractional colorings of $G$ is the fractional chromatic number $\chi_{f}(G)$. If instead we minimize over all $\{0,1\}$ valued fractional colorings we obtain the usual chromatic number $\chi(G)$, and thus $\chi_{f}(G) \leq \chi(G)$.

The Kneser graph $K_{a: b}$ has as its vertices the $b$-element subsets of an $a$-element set, where two vertices are adjacent if the sets are disjoint. For $a \geq 2 b$ it is well-known that $\chi_{f}\left(K_{a: b}\right)=a / b$ (see [21]), and Lovász [15] proved that $\chi\left(K_{a: b}\right)=a-2 b+2$.

A basic result is that for any $n$-vertex graph $G$ with independence number $\alpha(G)$, $\chi_{f}(G) \geq n / \alpha(G)$, and equality is achieved for example when $G$ is vertex-transitive, see [21]. We also have that $\chi_{f}(G) \geq \omega(G)$ and equality is achieved for all graphs $G$ with $\chi(G)=\omega(G)$ such as bipartite graphs and other perfect graphs. Finally, we observe that, if $H_{1}, H_{2}$ are graphs such that $H_{1} \cap H_{2}$ is a clique, then $\chi_{f}\left(H_{1} \cup H_{2}\right)=$ $\max \left\{\chi_{f}\left(H_{1}\right), \chi_{f}\left(H_{2}\right)\right\}$. Thus a fraction-critical graph has no separating set that is a clique.

The fractional gap of a graph $G$ is the quantity $\operatorname{gap}(G)=\chi(G)-\chi_{f}(G)$. For some families of graphs this gap can be linear in terms of the number of vertices, and in that case it is interesting to study the relative fractional gap, $\overline{g a p}(G)=\frac{\chi(G)-\chi_{f}(G)}{|V(G)|}$. We give a few small examples to illustrate this concept.

Example 2.1. Since $C_{2 k+1}$ is vertex-transitive we obtain $\chi_{f}\left(C_{2 k+1}\right)=\frac{2 k+1}{k}$, and thus $\operatorname{gap}\left(C_{2 k+1}\right)=\frac{k-1}{k}$ and $\overline{\operatorname{gap}}\left(C_{2 k+1}\right)=\frac{k-1}{2 k^{2}+k}$. Hence the odd cycle with the largest relative gap is $C_{5}$, namely $\overline{\operatorname{gap}}\left(C_{5}\right)=1 / 10$.

Perfect graphs have a gap of 0. Recall that the Strong Perfect Graph Theorem [3] says that a graph $G$ is perfect if and only if both $G$ and its complement $G^{c}$ do not contain an odd cycle on at least 5 vertices as an induced subgraph.

Example 2.2. The only graph on $n \leq 5$ vertices that has a positive gap is $C_{5}$ and $\operatorname{gap}\left(C_{5}\right)=1 / 2$.

A 6-vertex graph with a positive gap must contain an induced $C_{5}$ (otherwise it is a perfect graph) and either be 4-chromatic (that is a wheel $K_{1} \vee C_{5}$ ) or be triangle-free. In either case the gap is $1 / 2$.

Example 2.3. Larsen, Propp and Ullman [14] showed that if $M(G)$ is the graph obtained by applying Mycielski's construction to a graph $G$, then $\chi_{f}(M(G))=$ $\chi_{f}(G)+\frac{1}{\chi_{f}(G)}$. Thus for the triangle-free Grötzsch graph $M\left(C_{5}\right)$ we get $\chi_{f}\left(M\left(C_{5}\right)\right)=$ $\frac{5}{2}+\frac{2}{5}=2.9$ and $\chi\left(M\left(C_{5}\right)\right)=4$, so $\overline{\operatorname{gap}}\left(M\left(C_{5}\right)\right)=1 / 10$.

Example 2.4. Let $G$ be the graph on $n=7$ vertices obtained from two copies of the graph $K_{4}-u v$ by identifying the two copies of $u$ and joining the two copies of $v$ by an edge. $G$ is a 4 -critical graph known as the Moser spindle. It can be seen that $\chi_{f}(G)=3.5=n / \alpha(G)$, so that $\overline{g a p}(G)=1 / 14$.

Example 2.5. Consider $G$ with $V(G)=\{1,2, \ldots, 7\}$ whose edges form a 6-cycle on $1,2, \ldots, 6$ plus a triangle 246 and a $K_{1,3}$ with center 7 , and leaves $1,3,5$. Observe that $G$ is 4 -critical with $\alpha(G)=3$, which implies $\chi_{f}(G) \geq 7 / 3$. It can be seen that $\chi_{f}(G)=10 / 3$, and thus $\operatorname{gap}(G)=2 / 21$. 
Example 2.6. Let $M_{8}^{+}$be the nonplanar graph obtained from the Wagner graph/ Möbius ladder $M_{8}$ (that is the cycle on $1,2, \cdots, 8$ with added edges $15,26,37,48$ ) by adding vertex 9 adjacent to $1,3,5,7 . \chi_{f}(G)=3$, since $G$ contains triangles, and has a cover with the independent sets $928,946,136,358,572,714$ of weight $1 / 2 . M_{8}^{+}$is 4-critical, and $\overline{\operatorname{gap}}\left(M_{8}^{+}\right)=1 / 9$.

These examples and the fact that $\overline{\operatorname{gap}}(G)<1$ for all graphs $G$ motivate the following problem:

Problem 2.7. Determine $\bar{g}=\sup \{\overline{\operatorname{gap}}(G): G$ a graph $\}$.

Example 2.6 implies that $1 / 9 \leq \bar{g} \leq 1$, and this is the best lower bound construction we have. Corollary 2.13 below shows that $\bar{g} \leq 1 / 3$.

Observe that if $G$ is obtained as the join $H_{1} \vee H_{2}$ of two graphs $H_{1}$ and $H_{2}$ on $n_{1}, n_{2}$ vertices respectively, then $\chi(G)=\chi\left(H_{1}\right)+\chi\left(H_{2}\right)$ and $\chi_{f}(G)=\chi_{f}\left(H_{1}\right)+$ $\chi_{f}\left(H_{2}\right)$. Thus $\operatorname{gap}(G)=\operatorname{gap}\left(H_{1}\right)+\operatorname{gap}\left(H_{2}\right)$ and $\operatorname{gap}(G)=\frac{n_{1} \overline{\operatorname{gap}}\left(H_{1}\right)+n_{2} \overline{\operatorname{gap}}\left(H_{2}\right)}{n_{1}+n_{2}}$. We immediately obtain

Lemma 2.8. If $H_{1}, H_{2}$ are graphs with $\overline{\operatorname{gap}}\left(H_{1}\right) \leq \overline{\operatorname{gap}}\left(H_{2}\right)$, then $\overline{\operatorname{gap}}\left(H_{1}\right) \leq$ $\overline{\operatorname{gap}}\left(H_{1} \vee H_{2}\right) \leq \overline{\operatorname{gap}}\left(H_{2}\right)$.

Lemma 2.8 shows that every $\overline{\operatorname{gap}}(H) \in\{\overline{\operatorname{gap}}(G): G$ a graph $\}$ is achieved by infinitely many graphs, namely arbitrary joins of copies of $H$. The observation above also implies that this set is dense in $[0, \bar{g}]$.

Conjecture 2.9. There are graphs $G$ with $\overline{\operatorname{gap}}(G)=\bar{g}$.

More generally, we can define the maximum relative gap of a family of graphs $\mathcal{F}$ as $\sup \{\overline{\operatorname{gap}}(G): G \in \mathcal{F}\}$. The maximum relative gap need not be achieved by some $G \in \mathcal{F}$, but Conjecture 2.9 states that it is achieved when $\mathcal{F}$ is the family of all graphs. It would be interesting to find the maximum relative gap for vertex-transitive graphs, as this would tie the independence number of such graphs to their chromatic number. The following simple observation implies that the maximum relative gap for a family of graphs closed under taking subgraphs is determined by its color-critical graphs.

LEMMA 2.10. If $G$ has a nonempty vertex-set $S$ such that $\chi(G-S)=\chi(G)$, then $\overline{\operatorname{gap}}(G-S)>\overline{\operatorname{gap}}(G)$ unless $\operatorname{gap}(G)=\operatorname{gap}(G-S)=0$.

Combining Lemma 2.10 with Example 2.1 and the fact that every 2-colorable graph has gap zero we immediately obtain

Proposition 2.11. If $G$ is a 3-colorable graph, then $\overline{\operatorname{gap}}(G) \leq 1 / 10$ with equality only for $C_{5}$.

For more dense graphs we similarly get

Proposition 2.12. If $G$ is an n-vertex graph with $\alpha(G) \leq 2$, then $\operatorname{gap}(G) \leq n / 10$ with equality only for a join of some copies of $C_{5}$.

Proof. Let $G$ be a smallest counterexample. By Example 2.2 we may assume that $n>5$ and by Lemma 2.8 we may also assume that the complement $G^{c}$ of $G$ is connected.

Let $m$ be the size of a maximum matching in $G^{c}$. Observe that $\chi(G)=n-m$, since $\alpha(G) \leq 2$ implies that an optimal coloring uses $m$ color classes of size two and $n-2 m$ of size one. If $G^{c}$ has a perfect matching, then $m=n / 2$ and thus $\chi(G)=n / 2=n / \alpha(G)$. It follows that $\chi_{f}(G)=\chi(G)$ and the gap is zero. So we may assume that $G^{c}$ has no perfect matching. Thus by Tutte's 1-factor theorem there must be a vertex set $S$ such that in $G^{c}-S$ there are more than $|S|$ odd components, 
call this number $o\left(G^{c}-S\right)$. In fact the Tutte-Berge formula says that we can choose $S$ such that $2 m=n+|S|-o\left(G^{c}-S\right)$. Now in $G^{c}-S$ a largest matching is of size at $\operatorname{most}\left(n-|S|-o\left(G^{c}-S\right)\right) / 2=m-|S|$, so that $\chi(G-S) \geq(n-|S|)-(m-|S|)=\chi(G)$. Thus the result follows from Lemma 2.10 by induction unless $S=\emptyset$. In that case because $G^{c}$ is connected it follows that $o\left(G^{c}\right)=1$ and $2 m=n-1$. Since $n>5$ and $\frac{n+1}{2}=\chi(G) \geq \chi_{f}(G) \geq \frac{n}{2}$ we get that $\operatorname{gap}(G) \leq 1 / 2<n / 10$.

This now allows us to prove that $\bar{g} \leq 1 / 3$, to improve on the trivial $\bar{g} \leq 1$ :

Corollary 2.13. If $G$ is an $n$-vertex graph with $n \geq 3$, then $\operatorname{gap}(G) \leq \frac{n}{3}-1$.

Proof. For $n \leq 5$ the result follows from Example 2.2. For $n \geq 6$ proceed by induction. If $\alpha(G) \leq 2$, then the result follows from Proposition 2.12. Otherwise $G$ has an independent set $S$ of size 3 , and $\operatorname{gap}(G-S) \leq(n-|S|) / 3-1=n / 3-2$. However $\chi(G) \leq \chi(G-S)+1$ and $\chi_{f}(G) \geq \chi_{f}(G-S)$, so that $\operatorname{gap}(G) \leq \operatorname{gap}(G-S)+1 \leq$ $n / 3-1$, as desired.

3. Fractional coloring conjectures for planar graphs. We start this section by determining the maximum relative gap for planar graphs.

Proposition 3.1. If $G$ is a planar graph on $n$ vertices, then $\operatorname{gap}(G) \leq n / 10$.

Proof. By Proposition 2.11 and Lemma 2.10 a minimal counterexample $G$ to this proposition must be a 4-critical planar graph. By Grötzsch's theorem this implies that $G$ contains a triangle, and thus $\chi_{f}(G) \geq \omega(G) \geq 3$. (In fact $\omega(G)=3$ since otherwise $G$ has gap zero.) It follows that $\operatorname{gap}(G) \leq 1 \leq n / 10$ unless $n \leq 9$. By Example 2.2 it follows that $n \geq 7$. Toft [30] lists all 4 -critical planar graphs on at most 9 vertices, and it can be verified that for every such $G$ on $n=7,8,9$ vertices we have $\chi_{f}(G)>4-\frac{n}{10}$ : By Lemma 2.8 it follows that $G^{c}$ is connected which means we don't have to check odd wheels, and Examples 2.4 and 2.5 are the remaining graphs on 7 vertices. Toft's list contains one more planar graph on 8 vertices (with relative gap 1/12), as well as 14 planar (and 7 nonplanar) graphs on 9 vertices. If $G$ is a graph on 9 vertices with independence number 3 which contains some vertex not in an independent set of size 3 , then $\chi_{f}(G) \geq 10 / 3$ and thus $\overline{\operatorname{gap}}(G) \leq 2 / 27<1 / 10$. This proves the result for the planar graphs 1, 6, 16, 17, 19 and 20 from Toft's list. For the remaining 8 planar graphs on 9 vertices $(4,5,7$, and $11-15)$ it can be checked by computer that the largest relative gap is $4 / 45$, achieved by graphs 12 and 13 . To do so determine all maximal independent sets, and then the fractional chromatic number can be found by linear programming. We used the command linprog in MATLAB.

We believe that $C_{5}$ is the only graph with equality in Proposition 3.1. To prove this it suffices to check that for every planar 4-critical graph on 10 vertices $\chi_{f}(G)>3$. The following general conjecture would imply this.

Conjecture 3.2. If $G$ is a planar 4-chromatic graph, then $\chi_{f}(G)>3$.

Observe that the non-planar Kneser graphs $K_{2 b+2: b}$ are 4-chromatic but have $\chi_{f}\left(K_{2 b+2: b}\right)=2+\frac{2}{b}$. Perhaps somewhat surprisingly Conjecture 3.2 implies an old conjecture of Grötzsch that is stronger than the Four Color Theorem. To see this let $L(G)$ denote the line graph of a graph $G$ and recall that Tait proved that the Four Color Theorem is equivalent to the case $k=0$ in the following more general conjecture due to Grötzsch (see [12, 23]).

CONJECTURE 3.3. If $G$ is a planar 2-edge-connected graph of maximum degree 3 with exactly $k$ vertices of degree 2, then $\chi(L(G))=3$ unless $k=1$.

This conjecture follows immediately from Conjecture 3.2 by the next lemma. 
LEMma 3.4. If $G$ is a planar 2-edge-connected graph of maximum degree 3, then $\chi_{f}(L(G))=3$ unless $G$ has a unique vertex of degree 2 .

Proof. Observe that $\chi_{f}(L(G))$ is simply the fractional edge-chromatic number of $G$. The fractional edge-chromatic number (fractional index) is very well understood: By Theorem 4.2.1 in [21], it follows that $\chi_{f}(L(G))=\max \{\Delta(G), \Lambda(G)\}$, where $\Lambda(G)$ is the maximum value $\frac{2 e(H)}{n(H)-1}$ taken over all subgraphs $H$ of $G$ on an odd number of vertices. (This formula can be derived from Edmonds matching polytope [6], as shown by Lovász and Plummer [16]. This formula was perhaps formulated first by Seymour [22] who gave a different proof.) If $H$ has more than one vertex of degree at most 2 , then $2 e(H) \leq 3 n(H)-3$. If $H$ has a unique vertex $v$ of degree at most 2, then to avoid $v$ being incident to a cut-edge of $G$ it must have degree exactly 2 , and $G=H$. Thus $\Lambda(G) \leq 3=\Delta(G)$, unless $G$ has a unique vertex of degree 2 , in which case $\Lambda(G)=\frac{3 n-1}{n-1}>3$.

Next we state a few more interconnected conjectures, some of which may be hazardous.

Conjecture 3.5. A planar graph has fractional chromatic number 4 if and only if it contains a subgraph $H$ with independence number $\alpha(H)=|V(H)| / 4$.

CONJECTURE 3.6. There are only finitely many minimal planar graphs $G$ with independence number $\alpha(G)=|V(G)| / 4$.

CONJECTURE 3.7. There are only finitely many planar 4-fraction-critical graphs.

Remark 3.8. If Conjecture 3.5 holds, then ?? 3.6?? 3.7 are equivalent. One implication is immediate as every 4 -fraction-critical graph $G$ would need to be minimal with the property that $\alpha(G)=|V(G)| / 4$. Conversely, every minimal graph $G$ with $\alpha(G)=|V(G)| / 4$ contains a 4 -fraction-critical subgraph $H$ which in turn contains a subgraph $H^{\prime}$ with $\alpha\left(H^{\prime}\right)=\left|V\left(H^{\prime}\right)\right| / 4$, so that $G=H=H^{\prime}$ by minimality.

The only planar 4-fraction-critical graphs we know are listed in the following example.

Example 3.9. It can be checked that $K_{4}$ is the only 4-fraction-critical graph on fewer than 8 vertices.

The vertex-transitive planar graph $C_{8}^{2}$ has independence number 2 , and therefore $\chi_{f}\left(C_{8}^{2}\right)=8 / 2=4$.

The icosahedron $G_{12}$ is a vertex-transitive graph with $\alpha\left(G_{12}\right)=3$, and therefore $\chi_{f}\left(G_{12}\right)=12 / 3=4$.

Consider these two 16-vertex planar graphs $G_{16}$ with $\alpha\left(G_{16}\right)=4$, and therefore $\chi_{f}\left(G_{16}\right) \geq 16 / 4=4$ :

The first example is obtained from $C_{14}^{2}$ by inserting a vertex into each of the two faces of length 7 and making it adjacent to all the other vertices on this face. A second example is the 5-regular graph obtained from an 8-cycle $C=v_{1} v_{2} \ldots v_{8} v_{1}$, a 4-cycle $u_{1} u_{2} u_{3} u_{4}$ inside $C$ and a 4 -cycle $w_{1} w_{2} w_{3} w_{4}$ outside $C$ by joining each vertex of the 4-cycles to three consecutive vertices of the 8-cycles. (Here $u_{1}$ has neighbors $v_{1}, v_{2}, v_{3}$, and $w_{1}$ neighbors $v_{2}, v_{3}, v_{4}$.)

It can be verified that each of these 5 examples is 4 -fraction-critical.

The next observation shows that one way to prove Conjecture 3.7 would be to show that there is no sequence of planar graphs $H_{n}$ such that $\chi_{f}\left(H_{n}\right)$ is a strictly increasing sequence converging to 4 . (We call a graph family hereditary if it is closed under taking induced subgraphs.) 
Proposition 3.10. If a hereditary family of graphs $\mathcal{F}$ contains infinitely many $k$-fraction-critical graphs, then $k$ is an accumulation of fractional chromatic numbers of $\mathcal{F}$.

Proof. Let $G$ be a $k$-fraction-critical graph on $n$ vertices in $\mathcal{F}$. For each $v \in V(G)$ let $f_{v}$ be an optimal fractional coloring of $G-v$. By criticality $w\left(f_{v}\right)<k$. Moreover, $\frac{1}{n-1} \sum_{v \in V(G)} f_{v}$ is a fractional coloring of $G$. Hence it has total weight at least $k$. It follows that some $f_{v}$ must satisfy $k-\frac{k}{n} \leq w\left(f_{v}\right)<k$.

4. A general method and accumulation points for fractional chromatic numbers. We start with a simple observation.

Lemma 4.1. Let $G$ be a graph and $A_{1}, A_{2}, \ldots, A_{t}$ be vertex-sets so that every $v \in V(G)$ is contained in at most $s$ of these sets. If $\chi_{f}\left(G-A_{i}\right) \leq k$ for every $1 \leq i \leq t$, then $\chi_{f}(G) \leq \frac{t}{t-s} k$.

Proof. If $f_{i}$ is a fractional $k$-coloring of $G-A_{i}$, then $\frac{1}{t-s} \sum_{i=1}^{t} f_{i}$ is a fractional coloring of $G$.

This simple observation will be the key ingredient of the proofs in this section, as well as Theorem 5.2 in the next section. More immediately it also yields the following result.

Corollary 4.2. If $G$ is a $(k+1)$-vertex-critical graph on $n$ vertices, then

$\chi_{f}(G) \leq k+\frac{k}{n-1}$. Thus $\chi_{f}(G) \leq k+\frac{1}{2}$ unless $G=K_{k+1}$.

Proof. For each vertex $v_{i}$, let $A_{i}=\left\{v_{i}\right\}$. Applying Lemma 4.1 to $G$ with $t=n$ and $s=1$, we obtain a fractional coloring of size $\frac{n}{n-1} k$.

Gallai $[8,24]$ proved that if the complement $G^{c}$ of $G$ is connected, then $n \geq$ $2(k+1)-1$, and thus $\chi_{f}(G) \leq k+\frac{1}{2}$. Otherwise we may assume that $G^{c}$ has a component $G_{1}^{c}$ such that $G_{1}$ is not complete and is $\left(k_{1}+1\right)$-critical for some $k_{1}<k$. Then $\chi_{f}(G)=\chi_{f}\left(G-G_{1}\right)+\chi_{f}\left(G_{1}\right) \leq\left(k-k_{1}\right)+\left(k_{1}+\frac{1}{2}\right)$.

From Example 2.1 we see that the $(k+1)$-critical graph $G=C_{5} \vee K_{k-2}$ on $k+3$ vertices has $\chi_{f}(G)=k+1 / 2$. Combining Corollary 4.2 with Grötzsch's theorem we get the following.

CoROllary 4.3. If $G$ is a planar 4-critical graph, then $3 \leq \chi_{f}(G) \leq 3+\frac{3}{n-1}$, where the lower bound is always strict if and only if Conjecture 3.2 holds.

We now apply Lemma 4.1 to find some accumulation points of fractional chromatic numbers of planar graphs.

For context, observe that every rational number greater than 2 is the fractional chromatic number of some graph. Indeed, the Kneser graph $K_{a: b}$ (where $a>2 b$ ) has $\chi_{f}\left(K_{a: b}\right)=a / b$. However, for graphs on a fixed surface we do not know any interval in which the fractional chromatic numbers are dense. The odd cycles (respectively the odd wheels) show that there are sequences of fractional chromatic numbers of planar graphs converging to 2 (respectively 3) from above. Remark 6.6 in section 6 and Corollary 7.3 in section 7 show that there are planar triangle-free graphs with fractional chromatic numbers converging to 3 from below. The next result gives infinitely many more accumulation points.

Proposition 4.4. For every integer $m \geq 2$ the numbers $2+\frac{1}{m}$ and $3+\frac{1}{m}$ are accumulation points of fractional chromatic numbers of planar graphs.

Proof. Let $W_{n, m}$ be the graph obtained from a wheel on $n+1$ vertices by subdividing each spoke $m-1$ times. The graph $W_{n, m}$ has $n$ faces bounded by $(2 m+1)$ - 
cycles, and we let $W_{n, m}^{+}$be the graph obtained by inserting a vertex into each of these faces and making it adjacent to every vertex on the face. Observe that $\chi_{f}\left(W_{n, m}^{+}\right) \leq \chi_{f}\left(W_{n, m}\right)+1$, so that it suffices to prove that $\chi_{f}\left(W_{n, m}\right) \leq\left(2+\frac{1}{m}\right) \frac{n}{n-1}$ and $\chi_{f}\left(W_{n, m}^{+}\right)>3+\frac{1}{m}$ when $n$ is odd. (If $n$ is even then $W_{n, m}$ is homomorphic to $C_{2 m+1}$ and $W_{n, m}^{+}$to the odd wheel $W_{2 m+1,1}$. Thus $\chi_{f}\left(W_{n, m}^{+}\right)=\chi_{f}\left(W_{n, m}\right)+1=3+\frac{1}{m}$ so we restrict our attention to the case when $n$ is odd.)

Removing any of the $n$ vertices $v_{i}$ from the rim of $W_{n, m}$ we obtain a graph that is homomorphic to $C_{2 m+1}$, and thus $\chi_{f}\left(W_{n, m}-v_{i}\right)=2+\frac{1}{m}$. Applying Lemma 4.1 to $W_{n, m}$ where $A_{i}=\left\{v_{i}\right\}$ are the $t=n$ sets of rim vertices and $s=1$, we obtain $\chi_{f}\left(W_{n, m}\right) \leq \frac{n}{n-1}\left(2+\frac{1}{m}\right)$ as desired.

Now suppose that $f$ is an optimal fractional coloring of $W_{n, m}^{+}$when $n$ is odd. Then $f$ has weight sum at least $\chi_{f}\left(W_{2 m+1,1}\right)=3+\frac{1}{m}$. If it is precisely $3+\frac{1}{m}$, then $f$ induces an optimal coloring of each odd wheel $W_{2 m+1,1}$ in $W_{n, m}^{+}$and thus of each $(2 m+1)$-cycle in $W_{n, m}$. It follows that each independent set $I$ with $f(I)>0$ that meets one of the $(2 m+1)$-cycles must meet this $(2 m+1)$-cycle in exactly $m$ vertices. Since $m \geq 2$ it now follows that $I$ must meet every $(2 m+1)$-cycle in exactly $m$ vertices. Consider such an independent set $I$ containing the center $c$ of the wheel. Since $n$ is odd, then there must be a $(2 m+1)$-cycle $C$ such that $I$ does not contain the two vertices of $C$ at maximum distance from $c$. If $m$ is even, then $I$ cannot meet $C$ in $m$ vertices, a contradiction. If $m$ is odd, then we can argue similarly with an independent set $I$ not containing $c$. Thus for all odd $n$ we have $\chi_{f}\left(W_{n, m}^{+}\right)>3+\frac{1}{m} . \square$

Still the question if there is an interval for which the fractional chromatic numbers of graphs on some fixed surface is dense remains wide open. The graphs in Figures 6 and 7 in [19] suggest that there is an accumulation point that is between 11/4 and 4 , but not much else is known about accumulation points of fractional chromatic numbers of graphs on a fixed surface, even the plane. As a starting point we offer the following extension of Conjecture 3.7.

CONJECTURE 4.5. There is a constant $k$ such that for each fixed surface $\Sigma$, there are only finitely many fraction-critical graphs $G$ on $\Sigma$ with $\chi_{f}(G) \geq k$.

In [27] it was shown that for every surface $\Sigma$ there are only finitely many $\chi$-critical graphs $G$ with $\chi(G) \geq 6$ that embed on $\Sigma$. Perhaps $k=4$ will suffice in Conjecture 4.5. The only 4-fraction-critical graphs we know on the sphere are those in Example 3.9. Conjecture 4.5 (with $k=4$ ) implies the following 4-fractional-chromatic-conjecture:

CONJECTURE 4.6. For each surface $\Sigma$ there is a number $w_{\Sigma}$ such that any graph $G$ on $\Sigma$ with no noncontractible cycles of length at most $w_{\Sigma}$ has $\chi_{f}(G) \leq 4$.

The length of a shortest noncontractible cycle of a graph on a surface is called the edge-width of the embedding. The face-width is the smallest intersection the graph can have with some noncontractible curve on the surface. In [26] it was shown that large edge-width implies chromatic number at most 5, and that was extended by [27]. Thus possible counterexamples to Conjecture 4.6 must have large width and chromatic number precisely 5 . Two such classes of graphs are known, and below we verify Conjecture 4.6 for one of the classes.

The most general class are the Fisk triangulations, that is, triangulations with precisely two odd-degree vertices, and these vertices are neighbors. A Fisk triangulation can be realized on each surface, except the sphere, and such a triangulation has chromatic number at least 5 , see [18].

For the projective plane another class has been described by Mohar [17]. Youngs 
[31] made the surprising discovery that a nonbipartite quadrangulation of the projective plane has chromatic number precisely 4. Gimbel and Thomassen [9] proved that a triangle-free graph in the projective plane is 3-colorable unless it contains a nonbipartite quadrangulation. Mohar [17] added a vertex of degree 4 in each face of a quadrangulation $Q$ in the projective plane and proved that the chromatic number of the resulting graph $Q^{\prime}$ is either 3 (if $Q$ is bipartite) or 5 (if $Q$ is nonbipartite). Consider now the case where $Q^{\prime}$ (and hence also $Q$ ) has large edge-width. By Mohar's result, $Q^{\prime}$ has chromatic number 5 . By Theorem $5.2 \mathrm{~d}$ below, $Q$ has fractional chromatic number $2+\varepsilon$, and hence $Q^{\prime}$ has $\chi_{f}\left(Q^{\prime}\right) \leq 3+\varepsilon$. In particular, $Q^{\prime}$ satisfies Conjecture 4.6.

We now comment on Fisk triangulations. There are Fisk triangulations $G$ of large width containing $C_{8}^{2}$, so that $\chi_{f}(G) \geq \chi_{f}\left(C_{8}^{2}\right)=4$. Therefore we cannot use the proof of Theorem 5.2 below to prove that every Fisk triangulation $G$ has $\chi_{f}(G) \leq 4$. To see that a Fisk triangulation may contain $C_{8}^{2}$, let $F$ denote any Fisk triangulation, and let $e=x y$ denote the edge joining the two vertices of odd degree. Then $F-e$ has a facial 4 -cycle $C_{1}$. Identify the outer face of $C_{8}^{2}$ with $C_{1}$ to obtain a graph in which every vertex has even degree and there is a unique 4 -face in the inside of $C_{8}^{2}$. Add any edge $u v$ to non-adjacent vertices on this 4 -face to get a triangulation with $u, v$ the only vertices of odd degree.

5. Graphs on surfaces. In this section we will use Lemma 4.1 to prove some bounds for graphs of large width on a general surface. Theorem 5.2 below is already known for orientable surfaces. Indeed, DeVos, Goddyn, Mohar, Vertigan, and Zhu [4] established the same inequalities even for the circular chromatic number (which is greater than or equal to the fractional chromatic number) of graphs on orientable surfaces. But, those inequalities do no hold for the circular chromatic number of graphs on nonorientable surfaces, whereas Theorem 5.2 below holds for nonorientable surfaces as well.

The following result of Robertson and Seymour [20] (see also Theorem 5.9.2 in [18]) will be crucial.

THEOREM 5.1. For every graph $H$ embedded on a surface $\Sigma$ there is an integer $f(H, \Sigma)$ such that every graph on $\Sigma$ with face-width at least $f(H, \Sigma)$ contains $H$ as a surface minor.

The main result in this section is

THEOREM 5.2. For every surface $\Sigma$ and $\varepsilon>0$, there is an integer $g(\Sigma, \varepsilon)$ so that if every noncontractible cycle of a graph $G$ on $\Sigma$ has length at least $g(\Sigma, \varepsilon)$, then each of the following holds:

(a) $\chi_{f}(G)<4+\varepsilon$.

(b) If $G$ is an Eulerian triangulation, then $\chi_{f}(G)<3+\varepsilon$.

(c) If $G$ is triangle-free, then $\chi_{f}(G)<3+\varepsilon$.

(d) If every face of $G$ is even, then $\chi_{f}(G)<2+\varepsilon$.

Before we give the proof we would like to mention that these results are in some sense best possible. For (d) it suffices to observe that a sufficiently long noncontractible odd cycle can be extended to a graph in which each face is even in many ways. Theorem 5.3 below shows that (b) is best possible. For (a) we observe that any graph that contains $K_{4}$ will have $\chi_{f}(G) \geq 4$, but Conjecture 4.6 proposes that we can replace $\chi_{f}(G)<4+\varepsilon$ by $\chi_{f}(G) \leq 4$. We observe in Remark 6.6 in the next section that there are triangle-free planar graphs $G$ with $\chi_{f}(G) \geq 3-\frac{3}{n-1}$. Thus the $3+\varepsilon$ in (c) cannot be replaced by $3-\varepsilon$ (with some fixed positive $\varepsilon$ ), but it may be 
possible to replace it by 3 .

Proof. We will apply Lemma 4.1 with $s=2$ and $t=2+\lceil 8 / \varepsilon\rceil$, so that $\frac{t}{t-s} k<k+\varepsilon$ for $k=2,3,4$ as appropriate for the three cases.

For every surface $\Sigma$ of Euler genus $e g>0$ we can find simple closed curves $C^{1}, C^{2}, \ldots, C^{e g}$ such that $\Sigma-C^{1} \cup C^{2} \cup \cdots \cup C^{e g}$ is homeomorphic to a disc. We may assume that these curves are chosen so that there is no point contained in more than two of them. Let $H_{1}$ be the 4-regular graph on $\Sigma$ whose vertices are the intersection points of the $C^{p}$ and whose edges are the segments on these curves that connect them. (See Figure 5.1.) For $t \geq 2$ replace each $C^{p}$ by $t$ parallel copies $C_{1}^{p}, C_{2}^{p}, \ldots, C_{t}^{p}$ near enough to $C^{p}$ so that $C_{i}^{p}$ and $C_{j}^{q}$ only intersect if $C^{p}$ and $C^{q}$ intersect nearby. Finally let $H_{t}^{\prime}$ be the graph obtained from $H_{t}$ by replacing each degree 4 vertex by two degree 3 vertices as shown in Figure 5.1.
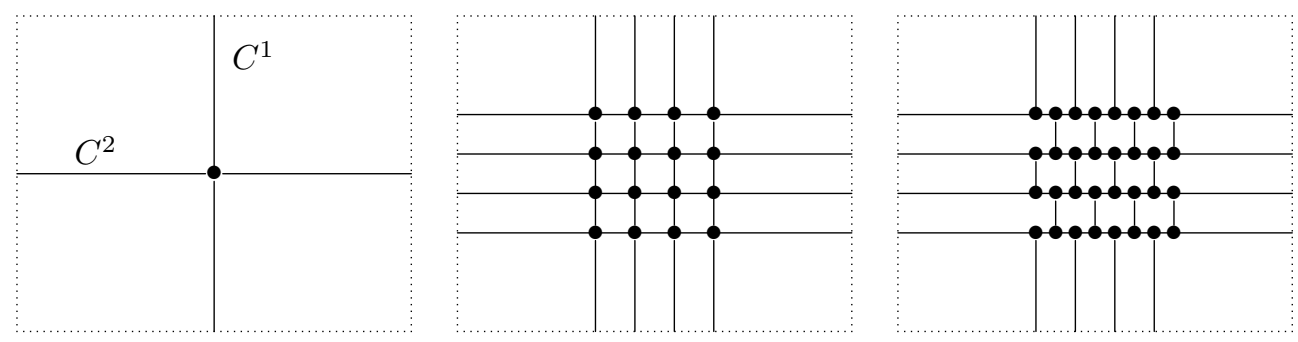

FIG. 5.1. $H_{1}, H_{4}$ and $H_{4}^{\prime}$ on the torus

Now we let $g(\Sigma, \varepsilon)=f\left(H_{t}^{\prime}, \Sigma\right)$. Thus if $G$ is a graph of face-width at least least $g(\Sigma, \varepsilon)$, then it contains $H_{t}^{\prime}$ as a surface minor and thus as a subdivision since $H_{t}^{\prime}$ is cubic. (Note that Theorem 5.2 assumes large edge-width whereas Theorem 5.1 requires large face-width. But, every graph $G$ of large edge-width can be extended to a graph $G^{\prime}$ of the same face-width. Moreover, if $G$ is triangle-free or has all faceboundaries of even size, then the extension can be made so that $G^{\prime}$ also has that property.) Let $A_{i}^{p}$ be the set of vertices in $G$ contained in the subdivision of $H_{t}^{\prime}$ that correspond to vertices on the curve $C_{i}^{p}$ in $H_{t}$. Thus $A_{i}=A_{i}^{1} \cup A_{i}^{2} \cup \cdots \cup A_{i}^{e g}$ has the properties that every vertex in $G$ is in at most two sets $A_{i}$, and the components of $G-A_{i}$ are planar.

Now (a) follows directly from the Four Color Theorem, and (c) from Grötzsch's theorem stating that planar triangle-free graphs are 3-colorable. For (d) observe that all faces of the components of $G-A_{i}$ must be even, except possibly the outer face, but it now follows easily that $G-A_{i}$ is bipartite. For (b) we observe that every Eulerian triangulation of the plane is 3-colorable. Every component $H$ of $G-A_{i}$ has the property that every face is a triangle (except possibly the exterior face) and that every vertex has even degree (except possibly some of those on the exterior face). It suffices thus to extend $H$ to an Eulerian planar triangulation, and we can do so by using two copies $H_{1}, H_{2}$ of $H$ and identifying each vertex in the exterior face of $H_{1}$ with its twin in $\mathrm{H}_{2}$.

It would be interesting to find a proof of (a), even for planar graphs, that does not require the Four Color Theorem. We now prove that the $\varepsilon$ in (b) cannot be omitted.

THEOREM 5.3. If $G$ is a triangulation of a surface, then $\chi_{f}(G)=3$ if and only if $\chi(G)=3$.

Proof. The backward implication is obvious, so suppose $\chi_{f}(G)=3$. Let $f$ be a 
fractional coloring such that $w(f)=3$. We call $f(I)$ the weight of $I$. It is easy to see that

(1) each independent set $I$ with positive weight contains precisely one vertex from each triangle $T$.

Let $v$ be a vertex and $I$ an independent set not containing $v$. Then (1) implies

(2) $I$ contains every second vertex in the neighborhood of $v$. In particular, $v$ has even degree.

Now let $I_{1}, I_{2}$ be two independent sets of positive weight containing $v$. Then $I_{1}, I_{2}$ contains no vertex in the neighborhood of $v$. Let $u$ be a neighbor of $v$. Applying (2) to $u$ and each of $I_{1}, I_{2}$ we conclude that $I_{1}, I_{2}$ agree in the neighborhood of $u$. In particular, $I_{1}, I_{2}$ agree on the second neighborhood of $v$. Repeating the argument, we conclude that $I_{1}=I_{2}$. So,

(3) there is only one independent set with positive weight containing $v$.

Let $T$ be a triangle. For each vertex of $T$, there is only one independent set with positive weight containing that vertex, by (3). Since every independent set with positive weight intersects $T$, we conclude that there are only 3 independent sets with positive weight. Those 3 independent sets give a proper 3 -coloring.

6. A method for degenerate graphs. Degeneracy arguments often yield fast coloring procedures. The fact that $K_{k}$ is a $(k-1)$-degenerate graph with $\chi_{f}\left(K_{k}\right)=k$ shows that the following result is in some sense best-possible. Remark 6.6 below will show that it is best possible in an even stronger sense.

Theorem 6.1. For $k \geq 2$, every $(k-1)$-degenerate $K_{k}$-free graph $G$ has $\chi_{f}(G)<$ $k$.

The following lemma will be a key idea in the proof of Theorem 6.1.

Lemma 6.2. For $k \in[2, \infty)$, every graph $G$ with $\chi_{f}(G)<k$ has a fractional coloring $f$ such that

(i) $w(f)<k$;

(ii) every independent (possibly empty) set I has positive weight $f(I)>0$; and

(iii) every vertex $v$ has total weight $w_{f}(v)=1$.

Proof. We proceed by induction on $n=|V(G)|$.

Let $\mathcal{I}$ denote the collection of independent sets of $G$, and let $i: \mathcal{I} \rightarrow[0, \infty)$ be the constant function with $i(I)=1$ for every $I \in \mathcal{I}$. If $g$ is any optimal fractional coloring, then we can find a small $\varepsilon>0$ such that $w(g+\varepsilon \cdot i)<k$. Thus $f_{0}=g+\varepsilon \cdot i$ is a fractional coloring of $G$ that satisfies (i), (ii).

Now let $V(G)=\left\{v_{i}: 1 \leq i \leq n\right\}$. If $n=1$ we define $f\left(\left\{v_{1}\right\}\right)=1$ and $f(\emptyset)=1 / 2$ to obtain the desired $f$. So assume that $n \geq 2$. By the induction hypothesis, let $c_{i}$ be a fractional coloring of $G-v_{i}$ satisfying (i),(ii),(iii) for each $i$ with $1 \leq i \leq n$. For each $i$ with $1 \leq i \leq n$, let $f_{i}$ be the fractional coloring defined by

$$
f_{i}=\frac{1}{w_{f_{i-1}}\left(v_{i}\right)} \cdot f_{i-1}+\left(1-\frac{1}{w_{f_{i-1}}\left(v_{i}\right)}\right) \cdot c_{i}
$$

Each $f_{i}$ satisfies (i), (ii) and $w_{f_{i}}(v) \geq 1$ for all $v \in V(G)$. Moreover $w_{f_{i}}\left(v_{i}\right)=1$ and it follows that $w_{f_{i-1}}(v)=1$ implies $w_{f_{i}}(v)=1$ for any $v \neq v_{i}$. Thus we conclude that $w_{f_{n}}(v)=1$ for all $v \in V(G)$. So $f=f_{n}$ satisfies (i)-(iii).

We will now prove Theorem 6.1.

Proof. We proceed by induction on $n=|V(G)|$, where the base case $n=1$ is obvious. So suppose that $n \geq 2$ and let $x \in V(G)$ with $d(x) \leq k-1$. By hypothesis, 
$\chi_{f}(G-x)<k$, so that by Lemma $6.2 G-x$ has a fractional coloring $f_{1}$ that satisfies Lemma 6.2 (i)-(iii).

Let $\mathcal{I}$ be the collection of independent sets of $G-x$ and $\mathcal{I}_{0}$ be the collection of those independent sets of $G-x$ (and thus $G$ ) that contain a neighbor of $x$ in $G$. By Lemma 6.2 (iii) and by the fact $d(x) \leq k-1$, we obtain

$$
\sum_{J \in \mathcal{I}_{0}} f_{1}(J) \leq \sum_{y \in N(x)} w_{f_{1}}(y) \leq k-1
$$

By Lemma 6.2 (ii), the first inequality is strict unless $N(x)$ is a clique of $G$; and the second inequality is strict unless $d(x)=k-1$. Since $G$ is $K_{k}$-free, one of the two inequalities must be strict, that is

$$
\sum_{J \in \mathcal{I}_{0}} f_{1}(J)<k-1
$$

Now we define a fractional coloring $f_{2}$ of $G$ as follows: If $\sum_{J \in \mathcal{I} \backslash \mathcal{I}_{0}} f_{1}(J) \geq 1$, then

$$
f_{2}(I)= \begin{cases}f_{1}(I), & I \in \mathcal{I}_{0} \\ f_{1}(I \backslash\{x\}), & x \in I \\ 0, & \text { otherwise }\end{cases}
$$

If $\sum_{J \in \mathcal{I} \backslash \mathcal{I}_{0}} f_{1}(J)<1$, then

$$
f_{2}(I)= \begin{cases}f_{1}(I), & I \in \mathcal{I}_{0} \\ f_{1}(I \backslash\{x\}), & x \in I \neq\{x\} \\ f_{1}(\emptyset)+1-\sum_{J \in \mathcal{I} \backslash \mathcal{I}_{0}} f_{1}(J), & I=\{x\} \\ 0, & \text { otherwise }\end{cases}
$$

It is easy to verify that $w_{f_{2}}(v) \geq 1$ for every $v \in V(G)$. Furthermore $w\left(f_{2}\right)=$ $w\left(f_{1}\right)<k$ for the first case; and $w\left(f_{2}\right)=1+\sum_{J \in \mathcal{I}_{0}} f_{1}(J)<k$ for the second case. In any case we have $w\left(f_{2}\right)<k$.

The following simple corollary of Theorem 6.1 can also be derived from Lemma 7.1 below.

Corollary 6.3. Every $K_{4}$-free 3-degenerate planar graph $G$ has $\chi_{f}(G)<4$.

Combining this with the result of Thomassen [28] that the vertex-set of every planar graph can be decomposed into an independent set and a 3-degenerate graph, we get an easy proof of the following weakening of the Four Color Theorem due to Hilton, Rado and Scott [11].

Corollary 6.4. Every planar graph $G$ has $\chi_{f}(G)<5$.

Theorem 6.1 combined with the fact that $\chi_{f}(G) \geq n / \alpha(G)$ immediately applies the following result. $n / k$.

Corollary 6.5. For $k \geq 2$, every $(k-1)$-degenerate $K_{k}$-free graph $G$ has $\alpha(G)>$

Remark 6.6. To see that Theorem 6.1 and Corollary 6.5 are in some sense best possible, observe that the graphs given in Figure 5 of Pirnazar and Ullman [19] give a sequence of triangle-free planar graphs $G_{m}$ on $5+3 m$ vertices with independence number $2+m$. These graphs are easily seen to be 2 -degenerate and satisfy $\chi_{f}\left(G_{m}\right) \geq$ 
$3-\frac{1}{m+2}$. For $k \geq 3$ the graph $G_{m} \vee K_{k-3}$ is $(k-1)$-degenerate $K_{k}$-free on $n=3 m+k+2$ vertices with $\chi_{f}(G) \geq k-\frac{3}{n-k+4}$ so that Theorem 6.1 is essentially best possible. For $k=3 p \geq 3$ in Corollary 6.5 observe that the graph $H_{m, p}$ obtained by replacing every vertex of $G_{m}$ with a $K_{p}$ (where two vertices of $H_{m, p}$ are adjacent if the corresponding vertices in $G_{m}$ are) has $n=(3 m+5) p$ vertices and $\alpha\left(H_{m, p}\right)=m+2=\frac{n}{k}+\frac{1}{3}$. Recalling that $k=3 p$ it is easy to see that $H_{m, p}$ is $(k-1)$-degenerate and $K_{k}$-free (in fact, its clique number is only $2 p=2 k / 3)$, and $\alpha\left(H_{m, p}\right)=\frac{n}{k}+\frac{1}{3}$.

Observe that $G_{m} \vee K_{1}$ is not planar, and so it is an open question if in Corollary 6.3 we can replace $\chi_{f}(G)<4$ by $\chi_{f}(G)<4-\varepsilon$ for some fixed positive $\varepsilon$.

We do not know if the approach in this section can be applied to other graph families, but in the next section we give a more flexible method.

7. A method for planar graphs with few triangles. As mentioned earlier, Hilton, Rado and Scott [11] showed that the Five Color Theorem for planar graphs can be extended to show that planar graphs are fractionally $(<5)$-colorable. Also, Dvořák, Sereni, and Volec [5] showed that Grötzsch's theorem 3-color theorem for planar triangle-free graphs can be extended to show that planar triangle-free graphs are fractionally $(<3)$-colorable. The proof in [11] is based on the fact that, for every vertex $v$ in a planar graph $G, G$ has a 5-coloring such that the neighbors of $v$ have only three colors. The proof in [5] is based on the fact that, for every vertex $v$ of degree at most 4 in a planar triangle-free graph $G, G$ has a 3 -coloring such that the neighbors of $v$ have only one color. The purpose of this section is to point that we do not need many colorings of this type. (In fact, only one such coloring suffices.) This approach can potentially apply more broadly, even though our upper bounds on the fractional chromatic number are not as strong as those in [11], [5]. (In fact, for triangle-free planar graphs of maximum degree 4 , the bound in [5] is sharp.)

We call a graph $k$-ambiguous if it has two $k$-colorings that differ in exactly one vertex. All $(k-1)$-colorable graphs are $k$-ambiguous. Uniquely $k$-colorable graphs such as $K_{k}$ are not $k$-ambiguous. Recall that a graph family is hereditary if it is closed under taking induced subgraphs.

Lemma 7.1. If $\mathcal{F}$ is a hereditary family of graphs such that every $G \in \mathcal{F}$ without a separating clique is $k$-ambiguous, then $\chi_{f}(G)<k$ for all $G \in \mathcal{F}$.

Moreover, if for some fixed integer $c$ every graph in $\mathcal{F}$ on $c$ vertices is $(k-1)$ colorable, then $\chi_{f}(G) \leq k-2^{-(n-c)}$ for every $G \in \mathcal{F}$ on $n \geq c$ vertices.

Proof. The first statement will follow from the second by choosing $c=1$. So suppose $G$ is a counterexample to the second statement with smallest order $n$. Clearly $n>c$, and $G$ can have no separating clique, or we can proceed by induction. Let $u$ be a vertex of $G$ so that $G-u$ has a $k$-coloring that can be extended to $G$ in at least two ways. Thus we can cover $V(G)$ with independent sets $I_{1}, I_{2}, \ldots, I_{k}$, where we can assume that $u \in I_{1}$ and $u \in I_{2}$. Let $f_{1}$ be the fractional coloring of $G$ such that $f_{1}(I)=1$ for $I=I_{j}$ with $1 \leq j \leq k$ and $f_{1}(I)=0$ otherwise.

Let $f_{2}$ be a fractional coloring of $G-u$ with weight $k-2^{-(n-1-c)}$. Now $f=$ $\frac{1}{2} f_{1}+\frac{1}{2} f_{2}$ is a fractional coloring of $G$ of weight $\frac{1}{2} k+\frac{1}{2}\left(k-2^{-(n-1-c)}\right)=k-2^{-(n-c)} . \square$

We give three applications of this method. Since it follows from Euler's formula that every triangle-free planar graph has a vertex of degree at most 3, Theorem 7.2 below proves that planar triangle-free graphs are 3 -ambiguous. Theorem 7.2 can be derived from the result of Grünbaum [10] (see also Aksenov [1] and Borodin [2]) that 
every planar graph with at most three triangles is 3-colorable. This is implicitly contained in the proof of Theorem 1 of Steinberg and Tovey [25] and is extended to vertices of degree 4 by Dvořák, Sereni and Volec [5].

THEOREM 7.2. Let $G$ be a triangle-free planar graph and $u$ be a vertex of $G$ of degree at most 3. Then $G$ has a 3-coloring such that all neighbors of $u$ receive the same color.

Since the smallest 3 -chromatic triangle-free graph is $C_{5}$ we can let $c=4$ in Lemma 7.1.

COROLlaRY 7.3. Every triangle-free planar graph $G$ on $n \geq 4$ vertices has $\chi_{f}(G) \leq 3-2^{-(n-4)}<3$.

Observe that Example 3.9 shows that a planar graph without $K_{4}$ as a subgraph need not have fractional chromatic number strictly less than 4 , so this result does not extend in that direction. On the other hand Dvořák, Sereni, and Volec [5] proved that in fact $\chi_{f}(G) \leq 3-\frac{3}{3 n+1}$ when $G$ is planar triangle-free, which is essentially achieved by the construction mentioned in Remark 6.6. Using Theorem 6.2 in [29] instead of Theorem 7.2 we get:

THEOREM 7.4. For each surface $S$, there exists a natural number $k_{S}$ such that the following holds: If $G$ is a graph on $n \geq 4$ vertices with girth at least 5 that is embedded on the surface $S$ without noncontractible cycles of length at most $k_{S}$, then $\chi_{f}(G) \leq 3-2^{-(n-4)}<3$.

Proof. By Euler's formula it follows that every girth 5 graph on a surface of Euler genus $g$ has a vertex $u$ of degree at most 3 , say with neighbors $v_{1}, v_{2}, v_{3}$. (The proof when $u$ has degree 2 is similar.) For each $v_{i}$ create a new vertex $u_{i}$ and consider the graph $H$ obtained from $G-u$ by adding edges to create a new facial cycle $C_{1}$ : $u_{1}, v_{1}, u_{2}, v_{2}, u_{3}, v_{3}$. Consider the pre-coloring of $C_{1}$ given by $L\left(u_{i}\right)=1$ and $L\left(v_{i}\right)=2$ for all $1 \leq i \leq 3$, and observe that this coloring can be extended to a 3-coloring of $H$ if and only if $G$ has a 3-coloring in which all neighbors of $u$ receive the same color.

We now put $k_{S}=f(g, s, q, r, k)$ where $f(g, s, q, r, k)$ is defined in connection with Theorem 6.2 from [29], and where $q=1, k=6, r=0$ and $s=3 g+3 q$. Assume now that $G$ (and thus $H$ ) has no noncontractible cycle of length at most $k_{S}$. We claim that the coloring can be extended from $C_{1}$ to all of $H$. For suppose that otherwise. Then Theorem 6.2 from [29] implies that $H$ has a subgraph $H^{\prime}$ with at most $f(g, s, q, r, k)$ vertices such that the coloring of $C$ cannot be extended to $H^{\prime}$. As $H^{\prime}$ has no noncontractible cycle, $H^{\prime}$ is planar, but this contradicts Theorem 7.2.

Our third application of Lemma 7.1 is in the following degeneracy setting: We call a graph triangle-degenerate if every one of its subgraphs has fewer triangles than vertices. Observe that for any even $n$, the planar graph $C_{n}^{2}$ has exactly as many triangles as vertices, but any of its proper subgraphs is triangle-degenerate. Thus the next theorem implies that $C_{8}^{2}$ and $K_{4}=C_{4}^{2}$ are 4-fraction-critical.

Theorem 7.5. If $G$ is a triangle-degenerate planar graph, then $\chi_{f}(G)<4$.

Proof. It suffices to show that every graph $G$ in this family without a separating clique is 4 -ambiguous.

Suppose $u$ is a vertex of $G$ such that $N(u)$ contains at most 2 edges. Since $G$ has no separating triangles it follows that each of these edges connects consecutive neighbors of $u$ and we can partition $N(u)$ into two consecutive sets of neighbors $N_{1}, N_{2}$ such that each of them is an independent set. Let $G^{\prime}$ be the graph obtained from $G-u$ by contracting $N_{1}$ to a vertex $u_{1}$ and $N_{2}$ to a vertex $u_{2}$ which is adjacent to 
$u_{1}$. Since $G^{\prime}$ is planar it is 4-colorable, which yields the desired 4-coloring of $G-u$ that only uses two colors in $N(u)$.

So we may assume that every vertex $u$ has at least 3 edges in its neighborhood, and thus is in at least 3 triangles. Hence $G$ must have at least $\frac{1}{3}(3 n)=n$ triangles, contradicting the assumption that $G$ has fewer triangles than vertices.

Theorem 7.5 and Corollary 6.3 are of independent interest: The 3-degenerate $K_{4}$-free planar graph $P_{n-2} \vee K_{2}^{c}$ has $2 n-6$ triangles and is therefore not triangledegenerate for $n>6$. Similarly triangle-degenerate planar graphs need not be 3 degenerate: If $v_{i}$ denotes the $i$-th vertex on $C_{n}$, then for even $n \geq 14$, the planar graph $C_{n}^{2}-v_{1} v_{3}+v_{1} v_{9}+v_{3} v_{9}$ has minimum degree 4 , but is triangle-degenerate.

Corollary 7.6. If $G$ is a planar graph that is $K_{4}$-free and $\left(K_{1} \vee P_{4}\right)$-free, then $\chi_{f}(G)<4$.

Proof. By Theorem 7.5 it suffices to show that if $G$ has $t$ triangles (none of which are separating) and $n$ vertices, then $t<n$.

Suppose that there are $e$ edges, $s$ triangles that do not share an edge with any other triangle, and $d$ edges that are in two triangles. Since $G$ is planar $K_{4}$-free, $\left(K_{1} \vee P_{4}\right)$-free and has no separating triangle it follows that no triangle shares an edge with more than one other triangle. Thus the total number of triangles $t=s+2 d$ and $e \geq 3 s+5 d$, so that $5 t \leq 2 e$. Since a triangulation on $n$ vertices has $3 n-6$ edges and $2 n-4$ triangular faces (by Euler's formula), we can turn $G$ into a triangulation by adding $3 n-6-e$ edges, where each edge adds at most 2 triangular faces. Thus $2 n-4 \leq t+2(3 n-6-e)$, or equivalently $t \geq 2 e-4 n+8$.

Suppose now (reductio ad absurdum) that $t \geq n$ and hence $4 t \geq 4 n$. Combining this with $t \geq 2 e-4 n+8$, we get $5 t \geq 2 e+8$, a contradiction to the earlier inequality $5 t \leq 2 e$.

Remark 7.7. The planarity requirement in Theorem 7.5 and Corollary 7.6 is necessary, as we can obtain triangle-free graphs of arbitrarily high fractional chromatic number by repeatedly applying Mycielski's construction to $C_{5}$. (See also Example 2.3. In fact, the graphs of large girth and large chromatic number constructed by Erdös [7] also have large fractional chromatic number.) In $C_{8}^{2}$ every vertex has $P_{4}$ as a neighborhood, justifying the condition $K_{1} \vee P_{4}$ in Corollary 7.6.

\section{REFERENCES}

[1] V. A. Aksenov, The extension of a 3-coloring on planar graphs, Diskret. Analiz, (1974), pp. $3-19,84$.

[2] O. V. Borodin, A new proof of Grünbaum's 3 color theorem, Discrete Math., 169 (1997), pp. $177-183$.

[3] M. Chudnovsky, N. Robertson, P. Seymour, and R. Thomas, The strong perfect graph theorem, Ann. of Math. (2), 164 (2006), pp. 51-229.

[4] M. DeVos, L. Goddyn, B. Mohar, D. Vertigan, and X. Zhu, Coloring-flow duality of embedded graphs, Transactions of the Am. Math. Soc., 357 (2004), pp. 3993-4016.

[5] Z. Dvořák, J.-S. Sereni, And J. Volec, Fractional coloring of triangle-free planar graphs, Electron. J. Combin., 22 (2015), pp. 7, Paper 4.11.

[6] J. Edmonds, Maximum matching and a polyhedron with 0,1-vertices, J. Res. Natl. Bur. Stand., 69B (1965), pp. 125-130.

[7] P. ERDős, Graph theory and probability, Canad. J. Math., 11 (1959), pp. 34-38.

[8] T. Gallai, Kritische Graphen. II, Magyar Tud. Akad. Mat. Kutató Int. Közl., 8 (1963), pp. 373-395 (1964).

[9] J. Gimbel and C. Thomassen, Coloring graphs with fixed genus and girth, Trans. Amer. Math. Soc., 349 (1997), pp. 4555-4564.

[10] B. Grünbaum, Grötzsch's theorem on 3-colorings, Michigan Math. J., 10 (1963), pp. 303-310. 
[11] A. J. W. Hilton, R. Rado, And S. H. Scott, A $(<5)$-colour theorem for planar graphs, Bull. London Math. Soc., 5 (1973), pp. 302-306.

[12] T. R. Jensen And B. Toft, Graph coloring problems, Wiley-Interscience Series in Discrete Mathematics and Optimization, John Wiley \& Sons, Inc., New York, 1995. A WileyInterscience Publication.

[13] A. D. King, L. Lu, And X. Peng, A fractional analogue of Brooks' theorem, SIAM J. Discrete Math., 26 (2012), pp. 452-471.

[14] M. Larsen, J. Propp, And D. Ullman, The fractional chromatic number of Mycielski's graphs, J. Graph Theory, 19 (1995), pp. 411-416.

[15] L. LovÁsz, Kneser's conjecture, chromatic number, and homotopy, J. Combin. Theory Ser. A, 25 (1978), pp. 319-324.

[16] L. Lovász AND M. D. Plummer, Matching theory, vol. 121 of North-Holland Mathematics Studies, North-Holland Publishing Co., Amsterdam; North-Holland Publishing Co., Amsterdam, 1986. Annals of Discrete Mathematics, 29.

[17] B. Mohar, Quadrangulations and 5-critical graphs on the projective plane, in Topics in discrete mathematics, vol. 26 of Algorithms Combin., Springer, Berlin, 2006, pp. 565-580.

[18] B. Mohar and C. Thomassen, Graphs on surfaces, Johns Hopkins Studies in the Mathematical Sciences, Johns Hopkins University Press, Baltimore, MD, 2001.

[19] A. Pirnazar and D. H. Ullman, Girth and fractional chromatic number of planar graphs, J. Graph Theory, 39 (2002), pp. 201-217.

[20] N. Robertson and P. D. Seymour, Graph minors. VII. Disjoint paths on a surface, J. Combin. Theory Ser. B, 45 (1988), pp. 212-254.

[21] E. R. Scheinerman and D. H. Ullman, Fractional graph theory, Dover Publications, Inc., Mineola, NY, 2011. A rational approach to the theory of graphs, With a foreword by Claude Berge, Reprint of the 1997 original.

[22] P. D. Seymour, On multicolourings of cubic graphs, and conjectures of Fulkerson and Tutte, Proc. London Math. Soc. (3), 38 (1979), pp. 423-460.

[23] - On Tutte's extension of the four-colour problem, J. Combin. Theory Ser. B, 31 (1981), pp. $82-94$.

[24] M. StenLík, Critical graphs with connected complements, J. Combin. Theory Ser. B, 89 (2003), pp. 189-194.

[25] R. Steinberg and C. A. Tovey, Planar Ramsey numbers, J. Combin. Theory Ser. B, 59 (1993), pp. 288-296.

[26] C. Thomassen, Embeddings of graphs with no short noncontractible cycles, J. Combin. Theory Ser. B, 48 (1990), pp. 155-177.

[27] — Color-critical graphs on a fixed surface, J. Combin. Theory Ser. B, 70 (1997), pp. 67100.

[28] — Decomposing a planar graph into an independent set and a 3-degenerate graph, J. Combin. Theory Ser. B, 83 (2001), pp. 262-271.

[29] - The chromatic number of a graph of girth 5 on a fixed surface, J. Combin. Theory Ser. B, 87 (2003), pp. 38-71. Dedicated to Crispin St. J. A. Nash-Williams.

[30] B. TofT, On critical subgraphs of colour-critical graphs, Discrete Math., 7 (1974), pp. 377-392.

[31] D. A. Youngs, 4-chromatic projective graphs, J. Graph Theory, 21 (1996), pp. 219-227. 